\title{
La colaboración público-privada en sanidad: hasta dónde y cómo delimitar sus fronteras*
}

\author{
Ricard Meneu \\ Fundación Instituto de Investigación en Servicios de Salud \\ Rosa Urbanos \\ Universidad Complutense de Madrid
}

\section{Resumen}

El artículo revisa algunas áreas de colaboración entre sector público y privado en sanidad. En particular, las que conciernen a la formación continuada de los profesionales, la investigación en salud y la gestión de los servicios sanitarios. En las dos primeras se pone de manifiesto la necesidad de controlar los conflictos de interés para evitar interferencias indeseadas de la industria. En la última, y tras varias décadas de «experimentos», se subraya la imprescindible y urgente mejora de la gestión pública, que solo si es muy buena podrá sacar partido de las ventajas que ofrecen las distintas fórmulas de colaboración público-privada.

Palabras clave: política sanitaria, gestión sanitaria.

Clasificación JEL: H11, H51, I18.

\begin{abstract}
The text reviews some of the most important areas of public-private collaboration in health systems. Particularly, it focuses on continuing medical education, health research and health care management. Regarding medical education and health research, the paper points to the need of eradicating inappropriate interferences of industry through regulating conflicts of interest. With respect to management, the article underlines the imperative improvement of public management as a previous step to be able of benefiting from the potential profits of public-private formulae.
\end{abstract}

Keywords: health policy, health management.

JEL classification: H11, H51, I18.

\section{Introducción}

El ánimo de lucro supone un poderoso incentivo para los agentes económicos y es un indudable elemento dinamizador de las economías que estimula la innovación y la eficiencia. También en sanidad, donde la presencia del sector público es predominante en el entorno de los países desarrollados, el sector privado juega un papel muy importante como agente colaborador en distintos ámbitos, que incluyen

* Los autores agradecen los comentarios de Beatriz González a una versión inicial del texto y la financiación del Ministerio de Economía, Industria y Competitividad (Proyecto ECO2017-83771-C3-2-R, Encaje Público-Privado en Sanidad), del Programa Estatal de I+D+i orientada a los retos de la sociedad. 
la investigación en nuevas formas de tratar la enfermedad, la diseminación de conocimiento científico o la misma gestión de algunos servicios sanitarios. Sin embargo, la maximización del beneficio económico como objetivo principal de los agentes privados puede entrar en conflicto con la defensa del interés general. Este problema es particularmente importante en sanidad por dos motivos: el primero, la presencia constante del fenómeno de información imperfecta y asimétrica; el segundo, que la atención sanitaria está lejos de ser considerada un servicio cualquiera. Ninguno de estos dos puntos exige comentarios adicionales.

Todo ello explica que, pese a que puede resultar útil y/o necesario contar con la colaboración del sector privado para ofrecer una atención sanitaria integral que proteja eficazmente la salud y atienda asimismo a consideraciones de eficiencia, sea imprescindible delimitar las fronteras (y formas de relación) entre lo público y lo privado. El debate sobre esta cuestión es continuo, y está teñido de fuertes consideraciones ideológicas. Más allá de elementos subjetivos, la pretensión de este artículo es la de aportar evidencia relevante sobre los efectos que puede causar una defectuosa delimitación de las fronteras anteriormente aludidas o una perfunctoria regulación de las formas de relación entre ambos agentes. Dicho de otro modo, su objetivo es el de contribuir a «delinear» adecuadamente el edificio sanitario que, desde sus inicios, se ha venido construyendo como suma de público y privado.

Sin ánimo de examinar con detalle todos los ámbitos en los que podría analizarse «hasta dónde» debe llegar la colaboración entre sector público y privado y «cómo» debe articularse (según reza el título del artículo), el texto se concentrará en algunos aspectos que, por su relevancia y/o por la controversia que generan, pueden señalar las líneas de acción de los próximos años. En particular, se abordarán los temas relativos a la formación continuada de los profesionales sanitarios, la investigación en salud y la colaboración del sector privado en la gestión sanitaria a través de sus distintas fórmulas.

\section{La formación médica continuada en el Sistema Nacional de Salud}

Si en un sector puede decirse que es imprescindible que los profesionales actualicen periódicamente sus conocimientos, ese es el sanitario. El ritmo de las innovaciones tecnológicas, tanto diagnósticas como terapéuticas, es incesante y creciente, y la publicación de nueva evidencia sobre los efectos de las tecnologías ya existentes es abrumadora, decenas de miles de artículos anuales recogidos en PubMed. La formación continuada es, en consecuencia, una herramienta fundamental en la provisión de una atención de calidad a los pacientes.

La participación del sector privado en las actividades de formación continuada de los profesionales médicos ha sido tradicionalmente muy importante en España, como consecuencia tanto de la escasez de recursos públicos para organizar esas actividades como del legítimo interés de la industria por promocionar sus productos. 
Dicho interés, no obstante, puede chocar con la necesidad de proporcionar a los profesionales una formación objetiva e independiente.

Son diversos los trabajos que analizan la participación de la industria en la formación de los profesionales y señalan sus posibles consecuencias (entre otros, Relman, 2001; Katz et al., 2002; Peiró, 2009; Avros y Choudhry, 2010; Stamatakis et al., 2013). Por ejemplo, Katz et al. (2002) comparan los efectos de distintos grados de participación de la industria en las actividades de formación continuada, y comprueban que en aquellas actividades con apoyo directo de la industria, el rango de temas ofrecidos es menor que cuando los programas se diseñan de forma independiente. Por su parte, Avorn y Choudhry (2010) señalan cómo la presencia de sesgos inconscientes y factores emocionales puede causar, aunque de forma no premeditada, influencias indebidas de la industria en las actividades de formación de los profesionales, tal y como sustenta, por otra parte, la creciente evidencia en Economía del Comportamiento. Otros trabajos previos proporcionan evidencia adicional en esta misma línea, como el estudio de Bowman y Pearle (1988), que constata cómo las actividades formativas patrocinadas por el fabricante se asocian con aumentos en las tasas de prescripción de los medicamentos del patrocinador (entre otras cosas como consecuencia de que los efectos positivos del medicamento patrocinado se mencionan hasta tres veces más que los de los competidores). En definitiva, se trata de meros desarrollos de los trabajos clásicos de Camerer (1988) o Carmichael (1997) sobre la sistemática estrategia de generación de relaciones de reciprocidad.

Es importante comprobar, además, cómo los profesionales no suelen percibir los posibles sesgos que pueden derivarse de sus relaciones con la industria. Los trabajos de Steinman et al. (2001) y Choudhry et al. (2002) muestran que la proporción de médicos que cree que su relación con la industria ejerce algún tipo de influencia en sus recomendaciones suele ser muy bajo, aunque la percepción sobre la influencia que la industria ejerce en sus colegas es mucho más intensa. Estos sesgos de percepción deberían llamar la atención sobre la idea de que, como decía Salvador Peiró, «Nosotros somos los otros» (Peiró, 2009).

En España también hay trabajos que ilustran cómo los médicos tienden a subestimar la influencia de la industria en las pautas de prescripción propias, pese a la evidencia al respecto (Grup d'Ética SCMFIC, 2004; Garcés et al., 2010; González-Rubio et al., 2017). Hay que tener en cuenta que una parte importante de las relaciones entre los profesionales y la industria farmacéutica o de tecnologías sanitarias se manifiesta a través de las actividades de formación (inscripciones a congresos -particularmente para los médicos de hospitales- o material de formación continuada) (Moliner et al., 2009; González-Rubio et al., 2017). El trabajo de Garcés et al. (2010) constata que, pese a que el 59,7 por 100 de una muestra de encuestados no suele asistir a cursos organizados por la industria farmacéutica, cuando asisten a un congreso mayoritariamente lo hacen invitados por la industria (en un 60,3 por 100). Además, aceptar actividades de carácter formativo es considerada una actividad ética por un 74,3 por 100 de los encuestados. Este hecho contribuye a explicar que la 
mitad de los profesionales encuestados no reconozca problemas éticos de ningún tipo en su relación con la industria.

La influencia de los intereses comerciales no se produce únicamente en lo que estrictamente se conoce como formación continuada, sino que también afecta a la formación reglada de posgrado (e incluso se observa en la formación de grado, como indican Stamatakis et al., 2013). La regulación pública en este ámbito es (además de mal conocida) escasa, de manera que las normas aplicables descansan básicamente en iniciativas de autorregulación (promovidas por la propia industria o por sociedades científicas o profesionales). Es el caso del Código de Buenas Prácticas de la Industria Farmacéutica (Farmaindustria, 2016), que no se refiere, al menos de forma explícita, a las actividades de formación de los profesionales, sino a todos los ámbitos de relación entre la industria y los profesionales y organizaciones sanitarias, y particularmente de la regulación de las actividades de promoción de medicamentos de prescripción y el desarrollo de la investigación.

Sin duda constituye un avance que, aunque financiadas por la industria, las iniciativas de formación no puedan estar organizadas, diseñadas ni ejecutadas desde la defensa de los intereses comerciales para que reciban la acreditación oficial. El problema es que, como se ha comprobado, el alejamiento de la industria respecto de las actividades de formación continuada, aunque ayuda a controlarlo, no elimina por completo el riesgo de sesgo (Josiah Macy Junior Foundation, 2008).

La abundante evidencia generada en este siglo explica el manifiesto suscrito por relevantes académicos señalando la necesidad de «una regulación más estricta, incluyendo la eliminación o modificación de prácticas comunes relacionadas con pequeños obsequios, formación médica continuada, financiación de viajes de médicos, autorías fantasma y contratos de consultoría e investigación» y proponiendo una política por la que los centros médicos académicos liderarían la eliminación de los conflictos de interés que aún caracterizan la relación entre los médicos y la industria de la atención sanitaria (Brennan et al., 2006). Esto es lo que ha ocurrido en algunas instituciones norteamericanas, entre las que se encuentran la Universidad de Michigan, el Memorial Sloan Kettering Cancer Center, la Academia de Médicos de Familia de Oregón, o incluso Kaiser Permanente en algunos estados de Estados Unidos, que han prohibido toda participación de la industria en la formación continuada (Spithoff, 2014).

Huelga decir que la definición del papel de la industria en la generación de conocimiento médico en general (y en la formación médica en particular), no está exenta de polémica. Incluso en instituciones tan reputadas como la Cochrane (organización sin ánimo de lucro dedicada a producir revisiones sistemáticas rigurosas sobre la evidencia disponible acerca de las intervenciones en salud), el grado de cercanía con la industria es una cuestión muy discutida, que ha derivado recientemente en la expulsión de uno de sus miembros más críticos. No obstante, la separación total entre «evidenciólogos» e industria ha sido apoyada públicamente por personajes tan influyentes como la editora del British Medical Journal (Godlee, 2018). 
En todo caso, en ausencia de una separación total entre las actividades de formación y los intereses comerciales, convendría que se abordara la regulación de los conflictos de interés desde una perspectiva que trascienda la mera voluntariedad, obligando a todos los profesionales a declarar públicamente sus relaciones con la industria (de la naturaleza que sean), incluyendo no solo las eufemísticas «transferencias de valor» o pagos directos, sino también cualquier tipo de actividad formativa subvencionada por la industria a la que haya asistido el profesional. La mejora de la transparencia constituye, tal y como se señala en algunos otros artículos de este mismo número (García-Altés y Ortún; Hernández) un elemento clave que las autoridades sanitarias deben promover con el fin de mejorar la gobernanza del Sistema Nacional de Salud. De forma complementaria, deberían potenciarse las habilidades de los médicos que, según ellos mismos, resultan críticas para mejorar su independencia, como son la lectura crítica o la búsqueda de evidencia rigurosa y fiable (Saito et al., 2014). Estas recomendaciones deberían extenderse a todos aquellos profesionales que puedan prescribir medicamentos (téngase en cuenta que el Real Decreto 1302/2018, de 22 de octubre, permite a los enfermeros recetar algunos fármacos). Pero, como ya se ha apuntado en otros textos (Meneu, 2010), por muy deseable que sea la generalización de códigos de buenas prácticas, la limitación, transparencia y los registros públicos de los obsequios recibidos por los sanitarios, todo quedará en una mera cosmética, un cambio a peor, si contribuimos a desplazar los lazos que inexcusablemente impone toda donación desde la mera gratitud por fruslerías accesorias hacia la obligada reciprocidad con quien patrocina $-y$ a menudo definenuestro mayor activo, la formación de los profesionales.

\section{La colaboración público-privada en investigación}

Las influencias de la industria que se han señalado en el epígrafe anterior también están presentes en lo que respecta a la investigación médica. Además, los conflictos de interés en este ámbito no siempre se hacen constar (sirva como ejemplo el escándalo del caso Baselga en fechas recientes), o se reportan de manera inadecuada (Roig y Borrego, 2017; Wayant et al., 2018).

Hay pruebas de que la participación del sector privado en investigaciones desarrolladas en centros y por profesionales públicos puede sesgar los resultados de diversas formas. Así, es sabido que los ensayos clínicos financiados por la industria tienden a ofrecer resultados alineados con sus intereses (Lexchin et al., 2003), lo que también ocurre con los estudios de coste-efectividad (Jørgensen et al., 2006), revisiones sistemáticas (Gøtzsche e Ioannidis, 2012) y meta-análisis (IOM, 2011). Este fenómeno se combina con el llamado «sesgo de publicación» (mayor probabilidad de publicación de los resultados positivos en las revistas científicas). Otra de las formas en las que la industria influye en la investigación que se publica está relacionada con lo que se conoce como «autoría fantasma» (ghost authorship), que consiste en incluir a investigadores de prestigio como primeros o segundos autores de ensayos 
clínicos que en realidad han sido escritos por personal de la compañía farmacéutica o por escritores médicos a sueldo de la misma (Stamatakis et al., 2013).

Por otra parte, y más allá de los sesgos relacionados con el desarrollo de los ensayos clínicos y la publicación de resultados, algunos autores han destacado que en los últimos años se ha acentuado el sesgo de la investigación hacia el logro de resultados patentables que puedan dar lugar a productos comercializables (Lüscher, 2012), lo que en última instancia tiende a generar aumentos en el gasto sanitario y dificulta la sostenibilidad de los sistemas de salud.

Esta posible tendencia a que los intereses del sector privado se impongan sobre los intereses generales (Novoa, 2015), incluso en las áreas donde se produce colaboración entre agentes públicos y privados, puede provocar importantes consecuencias. La lógica del ánimo de lucro implica, a priori, una relativa concentración de recursos en la búsqueda de tratamientos para las enfermedades más prevalentes, a costa de desatender relativamente, por ejemplo, la investigación en enfermedades de escasa incidencia y rentabilidad, o en tratamientos con un componente importante de externalidad (vacunas -salvo que proceda su aplicación a grupos amplios de poblacióno nuevos antibióticos) y, en todo caso, la investigación centrada en la prevención.

Incluso en el caso de las dolencias con mayor prevalencia, la investigación dirigida o patrocinada por la industria puede no corresponderse con la carga de enfermedad que se deriva de ellas. En un artículo muy reciente, Barrenho et al. (2018) comprueban que la innovación farmacéutica se concentra desproporcionadamente en las enfermedades cardiovasculares y circulatorias, el cáncer y las enfermedades muscoloesqueléticas. Asimismo, constatan que en los países desarrollados la innovación también se concentra desproporcionadamente en dolencias con menor carga de enfermedad.

Por otra parte, también se produce la paradoja de que el esfuerzo inversor de las administraciones públicas en investigación básica sea parasitado por la industria, que rentabiliza dichos esfuerzos mediante el registro de patentes que aprovechan el conocimiento científico financiado por fondos públicos, con apenas aportación de valor.

Algunos han denunciado un excesivo control por parte de la industria farmacéutica y de tecnologías de las distintas fases que forman la cadena del conocimiento biomédico: generación, difusión y aplicación (Novoa, 2015). Otros acusan al sector público, no tanto de descuidar cómo se formulan las reglas de colaboración público-privada en la investigación, sino de haber abandonado en gran medida el desarrollo de los ensayos clínicos en manos de la industria (Stamatakis et al., 2013). La reformulación de la «colaboración» entre sector público y privado en el ámbito de la investigación pasa por distintos frentes:

- Regular de forma estricta la obligatoriedad de que los autores hagan constar debidamente sus conflictos de intereses.

- Potenciar la investigación pública en aquellas áreas desatendidas por los intereses comerciales pero que tengan prioridad social, complementando además 
la investigación básica con investigación aplicada que impida que la industria capitalice la inversión financiada con fondos públicos.

- Contrarrestar los sesgos de la investigación financiada privadamente con el diseño de redes públicas globales que desarrollen una investigación objetiva e independiente, y que permitan aprovechar las sinergias que ofrece un mundo global en la generación y difusión del conocimiento, tal y como propone la llamada «Alianza para el buen gobierno del conocimiento biomédico» (Plataforma NoGracias, 2018).

En resumen, y en palabras de Ioannidis: «Debemos disminuir los sesgos, los conflictos de intereses y la fragmentación de los esfuerzos en favor de una investigación objetiva, transparente y colaborativa» (Ioannidis, 2015, p. 188).

\section{La colaboración público-privada en la gestión de los servicios sanitarios}

El debate sobre la colaboración entre lo público y lo privado en la gestión de los servicios sanitarios bascula esencialmente entre la defensa de la mayor microeficiencia que puede contribuir a alcanzar una mayor dosis de prestación privada, y los peores augurios sobre la capacidad de esta para distorsionar, esquilmar y degradar los principios de la financiación pública. Lo más descorazonador es que tanto los argumentos a favor como en contra parecen dar por sentado que los fallos empíricamente constatados del sector sanitario público -en administración, gestión de recursos humanos, regulación, pero quizás también en definición y supervisión de contratos- son consustanciales y no mitigables.

Conviene revisar el marco teórico y la evidencia que sustentan estos debates, así como revisar el campo de juego -la realidad de la colaboración entre sector público y privado en el sistema nacional de salud- teniendo presente que incluso en los dispositivos más fieramente públicos existen espacios tradicionalmente cedidos a proveedores privados.

Una taxonomía simple puede contemplar tres grandes métodos que resultan en la privatización de servicios y funciones previamente administrados por el sector público, sus organismos y empresas: 1) Delegación, donde lo público retiene la responsabilidad y la supervisión, pero utiliza el sector privado para la prestación de servicios, por ejemplo, mediante la contratación de servicios, o la subcontratación; 2) Desinversión, cuando la Administración Pública renuncia a la responsabilidad; y 3) Desplazamiento, en el que el sector privado crece hasta desplazar al sector público de una determinada actividad del gobierno (Savas, 1990). Cada una de estas formas incorpora varios enfoques específicos.

La prestación de asistencia sanitaria en todos los países implica algún tipo de colaboración público-privada. Incluso en países como España, donde la mayor parte de la asistencia se lleva a cabo directamente por el servicio público de salud, quedan ciertamente fuera de esa prestación pública la totalidad de las dispensaciones 
realizadas en oficinas de farmacia - una «privatización» nunca denunciada-, el transporte sanitario, prestado mayoritariamente con medios contratados, y buena parte de las diálisis, siendo también provistos por el sector privado los servicios suscritos mediante los clásicos conciertos «subsidiarios y complementarios» del INSALUD, tanto con clínicas y empresas comerciales, como los realizados con Cruz Roja, instituciones de la Asociación Española de Lucha contra el Cáncer (AELCC) y todo tipo de órdenes caritativas o asociaciones de pacientes.

En el ámbito de la atención primaria (AP), las experiencias de colaboración público-privada o de subcontratación están prácticamente ausentes, casi reducidas a la integración de esta asistencia en las concesiones administrativas y a la limitada experiencia catalana con una docena de entidades de base asociativa (EBA). Las EBA han de tener una entidad jurídica propia -generalmente son sociedades limitadas, pero caben las formas de sociedad anónima, sociedad laboral, sociedad laboral de responsabilidad limitada o cooperativa- constituidas total o mayoritariamente por profesionales sanitarios que establecen una relación contractual con el servicio público de salud para ofrecer servicios sanitarios a cambio de una financiación capitativa. Se trata de una de las formas de externalización sanitaria más específica, alineada con modelos prevalentes en los países de nuestro entorno y más reiteradamente evaluada (Síndic de Greuges, 2002; Fundación Avedis Donabedian, 2002; Institut d'Estudis de la Salud, 2006), pero curiosamente tiene un peso negligible, pues en el conjunto de la AP las EBA atienden a poco más del 3 por 100 de la población catalana.

De las diversas formas de colaboraciones público-privadas España ha tenido una larga tradición en el recurso a las figuras de los conciertos, que junto a los convenios singulares de vinculación eran las únicas previstas en la Ley General de Sanidad. Los conciertos más habituales incluyen la contratación externa de pruebas, diagnósticos y procedimientos terapéuticos para aliviar las listas de espera. También algunos de estos contratos cubren toda un área de población en áreas con infraestructura insuficiente (European Union, 2013).

A diferencia de lo señalado para la atención primaria, los centros privados tienen una importante contribución en la prestación de atención secundaria y especializada. Siguiendo la reciente revisión del European Observatory on Health Systems and Policies sobre el sistema sanitario español (Bernal-Delgado et al., 2018), a efectos de prestación asistencial de servicios financiados públicamente, los centros privados pueden actuar básicamente como:

- Prestadores subsidiarios de servicios, generalmente contratados para reducir listas de espera quirúrgicas.

- Red complementaria de la pública, como en el caso de la red de hospitales sin fines de lucro para uso público que produce una gran parte de la actividad en Cataluña, la llamada XHUP (Red de Hospitales de Utilización Pública).

- Proveedores sustitutos, cuya forma paradigmática es la de las concesiones administrativas a empresas privadas de la asistencia integral de departamentos/ áreas de salud completas en la Comunidad Valenciana, pero que también es el 
caso de camas de cuidado a largo plazo, generalmente de hospitales sin ánimo de lucro, utilizado en programas de cuidados paliativos o de alta temprana en pacientes crónicos.

Los conciertos entre los servicios públicos de salud y los operadores privados van más allá, en amplitud, de las reducciones de listas de espera quirúrgica, incluyendo un amplio elenco de actividades, como hospitalización, diagnóstico por la imagen, transporte sanitario, terapias respiratorias o diálisis. Aceptando los cálculos del IDIS (2018), un porcentaje significativo del gasto sanitario público (11,6 por 100) se destina a la partida presupuestaria de conciertos, con importantes variaciones entre CCAA. Cataluña es la comunidad autónoma que destina más dinero a esta partida, por un importe de 2.448 millones de euros, el 25,1 por 100 de su gasto sanitario, seguida por Madrid y Andalucía con 987 (12,4 por 100) y 398 (4,3 por 100) millones de euros, respectivamente.

Según la misma fuente, el 43 por 100 de los hospitales privados (193 hospitales) en España tiene suscrito algún tipo de concierto con las Administraciones Públicas, estando un 7 por 100 de aquellos (30 hospitales) integrados en la XHUP. Los que cuentan con un mayor número de conciertos son los hospitales generales, en un número de 122 hospitales, seguidos a distancia por los geriátricos, psiquiátricos o los médico-quirúrgicos.

El volumen económico de los conciertos con los hospitales privados no benéficos representó en 2016 el 26 por 100 de su facturación, una facturación de 1.578 millones de euros, de los que el 75 por 100 provenía de las comunidades autónomas y la cuarta parte restante de mutualidades de funcionarios y el sistema de Seguridad Social.

\subsection{Una escala de externalización (según su objeto)}

La forma prevalente de colaboración entre el sistema público de salud y el sector privado ha venido siendo la externalización, total o parcial, de diferentes tipos de servicios. Pero incluso empleando el mismo tipo de relaciones contractuales, de mecanismos de compra o de acuerdos concesionales, estas externalizaciones no tienen idéntica significación, en función del tipo de servicios afectados. Parece obvio que no tienen las mismas repercusiones ni en la integralidad de la prestación, ni en capacidad de captura, ni en afectación del «capital humano» del sistema público, la externalización de los servicios de lavandería que la del diagnóstico por imagen de alta tecnología (TAC, resonancia magnética, PET).

En el primer caso, seguramente cuesta justificar la conveniencia de una gestión autárquica de unas actividades básicamente coincidentes con las de diferentes sectores hosteleros, y que puede suponer importantes deseconomías de escala. En el segundo, extraer del acervo de conocimiento de la organización no solo la realización de las imágenes, sino su lectura, interpretación y las decisiones sobre la necesidad 
de exploraciones complementarias o adicionales, supone una descapitalización de un área nuclear de la asistencia sanitaria que fácilmente puede magnificar problemas de coordinación, especialmente cuando las distintas partes actúan bajo marcos de incentivos opuestos.

Hace una década, Repullo (2008) propuso cuatro situaciones de externalización de la provisión que afectan de modo diferente a la funcionalidad del servicio público: 1) externalización de centros, 2) externalización de servicios asistenciales, 3) externalización de servicios centrales, y 4) externalización de servicios generales. Mientras la primera se refería a la contratación con un centro privado de un amplio abanico de servicios sanitarios para una población protegida, la segunda era como la anterior pero limitada a unidades de servicios clínicos, la tercera se diferenciaba de la previa meramente en las funciones ancilares de las unidades subcontratadas (laboratorio, radiología, fisioterapia, etc.), circunscribiéndose la cuarta a servicios carentes de especificidad sanitaria, como cafetería, limpieza, lavandería, almacenes, etc.

Por conveniencia, para posteriormente poner en relación los diferentes tipos de servicios objeto de subcontratación con algunas consideraciones relevantes, reformularemos la tipología, invirtiendo además su gradiente, de menor a mayor especificidad.

Así, según sube el gradiente de especificidad tendríamos:

a) Externalización de servicios inespecíficos, generalmente de hostelería y logística.

b) Externalización complementaria de actividades de carácter clínico homologables y definidas (conciertos clásicos).

c) Externalización de procesos de elevada incertidumbre sobre su manejo e imprecisa definición.

d) Externalización plena de la actividad de unidades o servicios completos.

e) Externalización del conjunto de la asistencia reteniendo el aseguramiento.

La externalización de servicios inespecíficos $(a)$ se ha extendido ampliamente por el sistema sanitario, principalmente en las áreas de limpieza, lavandería y cocinas, alcanzando a menudo servicios que previamente no tenían una provisión directa, como la seguridad o algunos aparcamientos. Estos servicios suelen reunir las condiciones para un desplazamiento de proveedores con escasas o nulas repercusiones para la organización y con potenciales ganancias de microeficiencia. Las indudables experiencias conflictivas o los ocasionales encarecimientos son verosímilmente resultado de diseños o ejecuciones de contratos torpes o torticeros.

Un caso límite son los servicios de transporte sanitario, que dependiendo de su amplitud pueden incorporar especificidades sanitarias, lo que puede limitar los beneficios de la competencia en un ámbito territorial, al requerir de dotación específica sin suficiente uso alternativo. Aunque algunos estudios los etiquetan por su forma jurídica como «conciertos», en cuanto a las características de sus contenidos resulta preferible reservar ese término para los tipos siguientes. 
La externalización, con carácter complementario, de actividades asistenciales, diagnósticas o terapéuticas, en general con una definición neta de sus contenidos (b), ha sido la forma clásica de los conciertos que desde siempre ha suscrito el sistema público. El ejemplo más prevalente es el envío a centros privados de volúmenes variables de pacientes en lista de espera para procedimientos de relativamente escasa variabilidad. Con el tiempo la técnica contractual se fue perfeccionando y la unidad de pago pasó de la demasiado inespecífica «estancia hospitalaria»-modulada por el tipo de hospital, en lugar de por el procedimiento remitido- a alguna forma de definición de los procesos concretos a atender y sus correspondientes tarifas (Peiró et al., 1993). En estos casos el sistema público retiene sus capacidades y competencias, limitándose a drenar atenciones que exceden sus límites de actividad autoimpuestos. También se contaban en este tipo de conciertos los clásicamente suscritos para la realización de nuevas técnicas aún de bajo uso mediante equipos materiales y profesionales del sector privado, como ocurrió sucesivamente con TAC, resonancias y hasta PET, estas últimas ya en el marco de una pretendida evaluación de su «uso tutelado». De modo inverso al de los casos anteriores, en estos el sector público carecía del capital intelectual que proporcionaba el empleo de las nuevas tecnologías, pero no tanto por una «externalización» de algo que no había venido haciendo, como por una falta de «internalización» del nuevo know how y sus posibilidades de extensión.

En la externalización de procesos de elevada incertidumbre sobre su manejo e imprecisa definición $(c)$ se incluyen las remisiones, bajo forma de concierto, de casos en los que finalmente es el profesional subcontratado quien detenta las principales decisiones sobre las alternativas clínicas relevantes y que hacen mucho más impredecible y menos evaluable que en el caso anterior el contenido de la asistencia finalmente prestada. Con cierta frecuencia se da esta situación en la subcontratación de algunas cirugías, como la ortopédica, o allí donde se remiten a centros especializados o monográficos pacientes con diagnósticos oncológicos para su asistencia integral. No es el caso cuando esa remisión es para alguna asistencia pautada de modo explícito por una unidad clínica del dispositivo gestionado directamente -por ejemplo, 20 sesiones de radioterapia- que cabe confortablemente en el tipo anterior. Es a partir de aquí que empieza a desplazarse la «propiedad» del proceso, desde el prestador público al receptor en el centro privado, y con ello cuando empiezan las necesidades de mayores activos de conocimiento y especificidad para formular y evaluar las externalizaciones.

La externalización plena de la actividad de unidades o servicios completos $(d)$ es un escalón superior en la complejidad de la subcontratación, la pérdida de control sobre el proceso asistencial y la depleción de activos de conocimiento en la organización pública. Con diferencias de grado, estos efectos cabe esperarlos en subcontratación de servicios clínicos, pero también de servicios centrales, como laboratorio, diagnóstico por la imagen, farmacia, etc.

Finalmente, la externalización del conjunto de la asistencia (e) ha alcanzado gran relevancia en este siglo por la apuesta por concesiones administrativas territoriales 
en Valencia, con el llamado modelo Alzira, y su posterior extensión en Madrid, incrementando sucesivamente los contenidos de los previos contratos de PFI (concesión de obra pública). Pero este tipo ya tenía un precedente claro en los llamados «conciertos singulares»-convenio singular de vinculación-, que establecen la vinculación al sistema sanitario público de hospitales privados a través de la asignación de una población a atender.

En el caso de las concesiones tipo Alzira, se subcontrata la práctica totalidad de la asistencia sanitaria -suelen excluir farmacia ambulatoria, transporte sanitario, prótesis y otras prestaciones que financia directamente el servicio público-, por lo que al tratarse de una agrupación casi global de servicios el mecanismo de pago es capitativo, muy similar a una prima de seguro de salud pagada por el financiador público ${ }^{1}$. Conforme se avanza en este gradiente de especificidad de la externalización parece evidente que se requieren consideraciones más complejas sobre el objeto de la prestación, las especificidades sobre su gestión, el tipo y diseño de los contratos a suscribir, la evaluación de los resultados -asistenciales, más que económicos- de estos, las repercusiones sobre el conjunto de la organización, y sobre el conocimiento necesario para evaluar en el largo plazo la adecuación del funcionamiento del instrumental desplegado, las alternativas de rediseño y las posibilidades de vuelta atrás o reconfiguración de los acuerdos.

\subsection{Algunos principios generales a considerar en las decisiones sobre externalización}

Incluso si se omiten las consideraciones anteriores sobre las repercusiones a largo plazo de las decisiones de externalización, en función de algunos de los aspectos señalados, el comprador público debería sopesar las variables en juego: al menos, saber qué comprar -y definirlo y verificar su adecuada prestación-, decidir a quién comprarlo -las características de competencias, configuración, activos específicos, etc., que definen el tipo de proveedores más deseables- y luego valorar lo que ha comprado. Lo que supone ser capaz de preparar especificaciones cuidadosas adecuadas a lo que se va a comprar, emplear los mecanismos de compra competitiva en los casos en que pueda existir una competencia, así como disponer de criterios y mecanismos para monitorizar el desempeño del contratista.

Por tanto, hasta los manuales más practicones identifican una amplia serie de cuestiones que deben ser consideradas para cada alternativa, y entre ellas (Kelman, 2002): a) el tipo de contrato, en cuanto a su objeto, modalidades estructura de precios, escalamiento, etc.; $b$ ) selección de candidatos, identificando los servicios adecuados

\footnotetext{
${ }^{1}$ Esto ha llevado a algunas impugnaciones del modelo, al entender que supone una quiebra efectiva de la unidad de aseguramiento. Siendo una cuestión de complejos matices, no deja de sorprender que se plantee para un pretendido «aseguramiento» territorial, y no en lo que respecta a las mutuas (MUFACE, MUGEJU e ISFAS), en las que varios millones de ciudadanos experimentan una cesión similar de su responsabilidad asistencial.
} 
para la subcontratación; $c$ ) selección de fuentes, determinando si el proveedor debe ser único, actuar en competencia limitada o con competencia abierta; $d$ ) incentivos y modificaciones contractuales, así como mecanismos eficaces de resolución de reclamaciones y disputas, no la mera referencia a la legislación sobre contratos de las administraciones públicas; $e$ ) criterios para evaluar las ofertas, más allá de los rutinarios baremos de solvencia, experiencia, etc.; $f$ ) grado de discrecionalidad admisible, y $g$ ) naturaleza y grado de supervisión, algo demasiado habitualmente ausente de los diseños de licitación.

Un problema contractual crítico cuando se trata de servicios clínicos es la presencia y el cumplimiento de estándares de desempeño y calidad que deben respetarse, a veces incluso relacionados con pagos o sanciones. Según algunos análisis de las colaboraciones público-privadas europeas, es este uno de los aspectos en los que el sector público se esfuerza más por monitorizar y lograr el cumplimiento durante el periodo contractual. No es inusual que para esta parte importante de la gestión de contratos estén involucrados o sean necesarios agencias especiales, oficinas específicas o terceros (European Union, 2013). Sin embargo, la escasamente analizada experiencia española en este sentido muestra una extendida carencia de previsiones sobre evaluación del desempeño, sistemas de sanciones y una escasísima actividad de supervisión (Síndic de Comptes, 2018). Obviamente todas estas carencias son imputables a la administración que contrata, aunque no basta con señalar quién es responsable, sino también cuáles son las causas últimas y, por tanto, cómo se podrían superar.

La experiencia internacional acumulada permite ya apuntar que es verosímil que la subcontratación resulte más deseable que la provisión directa interna cuando se dan determinadas circunstancias. A partir de Kelman (2002) se pueden esquematizar algunas de las principales: 1) cuanto más precisa sea una tarea o resultado que se puede especificar por adelantado; 2) cuanto más fácilmente se puede medir y evaluar el rendimiento; 3 ) cuanta más competencia haya entre los posibles proveedores; 4) cuanto menos importante sea la actividad en la misión de la institución pública; 5) cuanto más variable sea la demanda de servicio a lo largo del tiempo; 6) si los proveedores privados pueden contratar personal capacitado con las habilidades necesarias más fácilmente que el prestador público; 7) si los proveedores privados tienen mayores economías de escala en la producción del servicio.

Respecto a la precisión en la especificación de las tareas, es obvio que es mucho más manejable describir un código diagnóstico o terapéutico para externalizar unos cuantos procesos, que definir el conjunto de la asistencia que debe prestarse a una población. Buena muestra de ello son los contratos de las concesiones administrativas que fijan como objeto «la cartera de servicios del SNS», con la previsible litigiosidad sobre si todo tipo de innovaciones, nuevos abordajes, o prestaciones emergentes están incluidas en tan asimétrico catálogo.

En cuanto a la facilidad para medir y evaluar el rendimiento, resulta inmediato apreciar que las especificaciones de estándares de los resultados de lavandería han debido normalizarse mucho más que las de, digamos, resultados clínicos tras una 
intervención quirúrgica. Y aun así, esa evaluación está mucho más respaldada que la valoración de los resultados poblacionales en salud, especialmente a la vista de los ingenuos indicadores de resultados que se siguen empleando en los análisis de eficiencia o en las memorias de salud, que insisten en esperanzas de vida o mortalidades mal ajustadas, cuando no retroceden a inferir los resultados a partir de la realización en porcentajes arbitrarios de algunos procesos.

Del grado de competencia entre proveedores se dispone de alguna evidencia empírica, especialmente gracias al informe de la Comisión Nacional de la Competencia (CNC) sobre los procesos de licitación para la provisión de la sanidad pública en España (CNC, 2013) en el que se identifican numerosos ejemplos de fracturas de la competencia efectiva, y se cuestionan muchas de las licitaciones analizadas entendiendo que cabe apreciar indicios de colusión.

La cautela sobre la importancia de la actividad externalizada en la misión de la institución pública parece aconsejar refrenar los impulsos de subcontratar la totalidad de algunas actividades clave y, de modo especial, de hacerlo territorialmente con el conjunto de actividades que constituyen en sí la misión de la institución. También recomienda atender a las relaciones entre los diferentes componentes de la prestación sanitaria ya que, por ejemplo, la compra de servicios de radiología o patología de un proveedor externo, a menudo con sede en un lugar diferente, separa al personal que informa los resultados de aquellos que prestan la atención. En esos casos la insuficiencia de contexto clínico o interacción suficiente puede disminuir la calidad de interpretación de casi todas las pruebas de diagnóstico.

Otras cautelas, como las fluctuaciones de demanda a lo largo del tiempo o la mayor facilidad para contratar personal capacitado resultan menos generalizables, siendo muy dependientes de especialidades, tamaño del mercado local, restricciones autoimpuestas por el prestador público en sus modos de contratación, etc.

Por su parte Machado y Crespo (2011), al revisar el estado del arte internacional de la externalización sanitaria, reconocen que las diferencias de contexto son cruciales para comprender las ventajas y los riesgos de la subcontratación en el marco de cada sistema de salud. Aun así aprecian que el nivel de subcontratación -tanto clínico como no clínico- disminuye a medida que aumenta el tamaño del hospital y que un patrón dominante es que se internalizan los servicios de atención directa al paciente. También que los riesgos más reportados de la subcontratación de actividades clínicas se refieren a las dificultades de integración, incluso en actividades como la radiología y otras funciones de laboratorio.

Otras consideraciones a tener en cuenta ya han sido apuntadas. A menudo se recurre al marco de la economía de los costes de transacción (ECT), iniciado por Oliver Williamson (1990), para explicar por qué es probable que las formas más intensas de colaboración público-privada se asocien con costos de transacción más altos que otras formas de prestación (Lonsdale, 2005). En un marco de racionalidad limitada y orientación hacia el interés propio de los actores, cualquiera de estos puede aprovechar las lagunas en el conocimiento del otro para promover sus intereses. El impacto de estos comportamientos en los resultados depende de dos dimensiones de 
la transacción: la especificidad de los activos y la incertidumbre, por ejemplo, sobre en qué medida los objetivos pueden ser cambiantes. Ante incertidumbres altas en una industria que cambia rápidamente como la atención de la salud, existe una gran probabilidad de que el contrato sea incompleto y que se necesiten renegociaciones durante su vigencia (Lonsdale 2005), lo que permite predecir que los procesos de negociación y monitorización de los contratos serán extensos e implicarán costes sustanciales (Vecchi y Hellowell, 2018).

Además, la especificidad de los activos y la escasez de competidores, mayor cuanto más amplia sea la externalización, suponen riesgos adicionales de captura para las partes. Ya se han apuntado las repercusiones sobre la integralidad de la prestación, pero también sobre el conjunto de la organización, que deja de trabajar en un marco común, debiendo adaptar las nuevas directrices para el conjunto de la red propia a las estipulaciones contractuales de los servicios externalizados, dualizando y duplicando sus tareas. A lo que hay que añadir la progresivamente manifiesta carencia de los conocimientos específicos necesarios para diseñar contratos no absolutamente incompletos, valorar su cumplimiento, ajustar en el largo plazo el funcionamiento de las herramientas desplegadas, formular alternativas de reconfiguración de los acuerdos y evaluar el logro de los objetivos pretendidos. Así, el balance de una externalización de toda una especialidad debe considerar no solo los ahorros obtenidos, sino también las pérdidas de conocimiento, integralidad de la prestación, afectación del «capital humano», competitividad profesional, etc., y el hecho de que a medio plazo impondrán importantes costes en el caso de decaer la subcontratación.

\subsection{Sobre la relativa eficiencia de la prestación pública y privada}

Buena parte de los debates sobre la prestación privada de servicios sanitarios públicos pone el acento en la mayor eficiencia mostrada por el sector privado, dando por generalmente compartido que a la ciudadanía le interesa recibir adecuadamente los servicios, con independencia de quién se los presta. Para ello se destacan los datos que pueden avalar que el sector privado resulta más eficiente, adoptando una parcial definición de la eficiencia. Así, para señalar que los servicios prestados privadamente son más eficientes se muestran meramente sus menores costes, asumiéndose un implícito ceteris paribus sobre los resultados. Pero sobre esos resultados, cuya equiparación permite determinar que su logro con menores costes supone mayor eficiencia, existen verdaderos océanos de falta de información. Los trabajos publicados que contemplan la calidad de los resultados son escasos y generalmente parciales.

Con todo, un mero repaso de la evidencia más reciente sobre ejecutoria comparativa de prestadores públicos y privados en el marco de la sanidad pública matiza bastantes de las afirmaciones usualmente esgrimidas por los partidarios de cualquiera de las modalidades. Así, aunque numerosas contribuciones teóricas relevantes sugieren que los hospitales privados deberían superar a los hospitales públicos en términos 
de eficiencia, la evidencia empírica en los mercados regulados y mixtos de atención sanitaria de Europa es mucho más diversa.

En una reciente revision de síntesis (Tynkkynen et al., 2018) se muestra que, aunque muchos estudios aportan resultados sin significación, la mayoría de los restantes encontraron que los hospitales públicos muestran mejor desempeño que los proveedores privados sin ánimo de lucro (PNFP), los que, a su vez, presentan un desempeño ligeramente mejor que los hospitales privados lucrativos en términos de medidas de eficiencia. Para ello se identificaron 24 estudios que informaron medidas de eficiencia económica o calidad en su comparación de organizaciones hospitalarias con diferentes formas de propiedad que cubrían una amplia gama de países europeos, incluyendo Austria, Alemania, Inglaterra, Francia, Grecia, Italia, España, Suiza y Noruega, representando a más de 5.500 hospitales en Europa.

Sin embargo estos resultados deben ser tomados con las razonables cautelas. El contexto es importante para entender estos resultados. Reiteradamente se ha señalado que los aspectos específicos del sistema de financiación, el proceso de contratación y el grado de competencia o monopolio en el mercado son factores importantes para determinar los efectos de la propiedad. En términos generales, parece probable que los resultados sean sensibles a las circunstancias específicas y la configuración regulatoria. Cabe pues especular que la variación en los resultados hallada entre países y en el tiempo puede explicarse en parte por las diferencias en los costes de transacción, la estructura y la madurez del mercado.

En otra revisión de este año, etiquetada por los propios autores como «realista», se compara el desempeño de los hospitales públicos con los hospitales privados en cuanto a eficiencia, accesibilidad y calidad de atención en la Unión Europea, sintetizando estudios de Italia, Alemania, Reino Unido, Francia, Grecia, Austria, España y Portugal (Kruse et al., 2018). La mayoría de la evidencia sugiere que los hospitales públicos son al menos tan eficientes o más que los hospitales privados. Con todo, se señala que la evidencia existente sobre la calidad de la atención a menudo es demasiado diversa para hacer una declaración concluyente.

En nuestro país ya disponemos de literatura que ha explorado las diferencias en eficiencia de los centros sanitarios del SNS atendiendo a sus características de propiedad y gestión. Los resultados del último de ellos (Pérez-Romero et al., 2017) indican que el hecho de disponer el hospital de personalidad jurídica, con independencia de su propiedad pública o privada, aumenta la eficiencia técnica en torno a 11,4 puntos respecto a los hospitales públicos tradicionales, y que el marco de regulación y gestión del hospital es más relevante que la propiedad pública o privada. Se postula que la dotación de personalidad jurídica al hospital del SNS supone someterlo a un marco jurídico y de gestión mucho más flexible que el que caracteriza a los hospitales tradicionales, por lo que se considera que es relevante que, en los hospitales analizados, la personalidad jurídica (pública o privada) se asocie a un marco de regulación de tipo laboral y no estatutario.

Algo similar venían mostrando los sucesivos informes de IASIST (2013), generalmente enarbolados como prueba de la superioridad de los centros privados, 
pero que en puridad reflejan el generalizado mejor desempeño de los hospitales con (otras) formas de gestión distintas a la gestión directa (OFG) respecto a los hospitales públicos de gestión directa administrativa (GDA). Frente a estos, los hospitales del grupo OFG, con modelo de gestión más flexible y autónomo, aparecían como claramente más eficientes y productivos, al igual que en estudios similares previos. Con todo, ese grupo estaba constituido mayoritariamente por hospitales acogidos a muy diversas configuraciones, y su comportamiento no impedía que algunos de los hospitales de modelo GDA alcanzaran resultados que los sitúan entre los más eficientes.

\section{Conclusiones}

De lo expuesto pueden derivarse algunas conclusiones, más o menos sintéticas, que se refieren a continuación:

1. La formación médica continuada, al igual que la investigación, tiene características de bien público. Ya sea por desinterés del mercado (investigación en comportamientos saludables que prevengan la enfermedad, por ejemplo), o bien por el riesgo de que el sector privado genere influencias indebidas en la generación y difusión del conocimiento médico, que finalmente se traducen en la práctica clínica (lo que implica que el mercado falla), el sector público tiene la obligación de intervenir. La regulación estricta de los conflictos de interés, que incluya una total transparencia de las relaciones entre la industria y los profesionales, contribuiría sin duda a alinear los intereses particulares con el interés general. Un aumento de la inversión pública en formación e investigación también sería deseable si se lograsen desviar los recursos necesarios a estas áreas, reduciendo, por ejemplo, las bolsas de ineficiencia existentes hoy por hoy en el sistema sanitario. El retorno que cabría esperar de estas actuaciones sería una mejor adecuación de la práctica clínica a las necesidades de los pacientes que podría favorecer la sostenibilidad del Sistema Nacional de Salud.

2. En lo que respecta a la gestión de los servicios sanitarios, es preciso insistir en explotar el aprendizaje que pueden aportar los experimentos a prueba de fallos con las más nuevas formas de colaboración público privado -concesiones, PFI-, a pesar del mínimo conocimiento obtenido respecto a sus efectos tras más de tres lustros «experimentando». Parece cierto que, en general, el regulador se ha movido de una forma de provisión a otra sin solución de continuidad y sin reclamar suficientemente la información deseable para monitorizar su buen curso. Aquí, sin duda, puede haber jugado también un apriorismo político, simétrico al rechazo generalizado (López-Casasnovas y del Llano, 2017).

3 . Sin duda hay razones comprensibles para querer perpetuar el diseño verosímilmente menos eficiente: una generalizada provisión bajo gestión admi- 
nistrativa directa. Algunas serán por «principios» (menor tolerancia social a la apropiación de excedentes cuando son cuantificables económicamente que cuando se expresa en baja productividad y costes unitarios altos, como parte de la ineficiencia de la administración) (López-Casasnovas y Del Llano, 2017). Otras, por interés (los actuales beneficiarios de esa apropiación). Pero también por prudencia (¿estamos seguros de mejorar cambiando las reglas del juego y manteniendo la alineación: profesionales entrenados en el campo de juego estatutario, administradores con déficits notables de formación, miras y experiencia en entorno competitivo, y gestores crecidos en las relaciones estatutarias y unas prácticas perpetuadas desde la administración colonial?).

4. Tras más de un cuarto de siglo discutiendo sobre las modalidades de prestación asistencial diferentes a la llevada a cabo directamente por la Administración Pública en el marco de las relaciones laborales «estatutarias» y el sometimiento al procedimentalismo administrativista con escasos avances en la evaluación y el conocimiento derivado de tantos «experimentos» $\mathrm{y}$ «modelos», va siendo hora de pensar en que la imprescindible mejora de la eficiencia y deseabilidad de los servicios sanitarios financiados públicamente afecten al núcleo del SNS, al conjunto común de sus instituciones.

5. Aunque omitidas en este texto, no se pueden desatender las llamadas formas de «privatización funcional» (Menéndez, 2008), consistentes en recurrir a mecanismos de gestión directa a través de entidades de titularidad pública sometidas a derecho privado, es decir, al conjunto de los organismos del sector público instrumental. La escasa evidencia disponible apunta su mejor logro, mejor en ocasiones que el de ciertas configuraciones privadas. Dada la multiplicidad de las alternativas ya experimentadas, sus características diferenciales o las consideraciones que pudieron aconsejar optar por unas u otras, deberían ser analizadas, junto a las distintas ejecutorias de cada diseño. Con esos mimbres cabría apostar informadamente por extender su uso en aquellas modalidades y prestaciones donde se estime que los beneficios superan el conjunto de costes relevantes.

6. Las respuestas a todo esto distan de ser únicas, pero se necesita de una (más que mejor) muy buena gestión pública para que funcionen tanto las formas más intensas de colaboración público-privada como las opciones más conservadoras o regresivas de republificar y reestatutarizar, en un retorno al pasado poco justificable para la sociedad del siglo Xxi. Hay prisa, porque estamos dejando pasar la ventana de oportunidad del rediseño que ofrece el mayor relevo de profesionales que hemos visto en nuestra historia. Estamos asistiendo a la jubilación de los contingentes de las oleadas históricas del crecimiento hospitalario y la extensión inicial del nuevo modelo de la AP en tiempos de escasa, si alguna, reposición (de ahí la precariedad). Pero nos hemos dado tanta prisa por tardar que aún no hemos desbrozado las líneas maestras de los posibles cursos de acción más deseables para el conjunto del sistema público, y no solo para «experimentos» que tienen lugar en su periferia. 


\section{Referencias bibliográficas}

[1] AVORN, J. y CHOUDHRY, N. K. (2010). «Funding for medical education: maintaining a healthy separation from industry». Circulation, 121 (20), 2228-2234.

[2] BARRENHO, E.; MIRALDO, M. y SMITH, P. C. (2018). «Does global drug innovation correspond to burden of disease? The neglected diseases in developed and developing countries». Health Economics, 1-21.

[3] BERNAL-DELGADO, E.; GARCÍA-ARMESTO, S.; OLIVA, J.; SÁNCHEZ MARTÍNEZ, F. I.; REPULLO, J. R.; PEÑA LONGOBARDO, L. M.; RIDAO-LÓPEZ, M. y HERNÁNDEZ-QUEVEDO, C. (2018). «Spain: Health system review». Health Systems in Transition, 20 (2), 1-179.

[4] BOWMAN, M. A. y PEARLE, D. L. (1988). «Changes in drug prescribing patterns related to commercial company funding of continuing medical education». Journal of Continuing Education in the Health Professions, 8, 13-20.

[5] BRENNAN, T. A.; ROTHMAN, D. J.; BLANK, L; BLUMENTHAL, D.; CHIMONAS, S. C.; COHEN, J. J.; GOLDMAN, J.; KASSIRER, J. P.; KIMBALL, H.; NAUGHTON, J. y SMELSER, N. (2006). «Health industry practices that create conflicts of interest: a policy proposal for academic medical centers». JAMA, 295, 429-433.

[6] CAMERER, C. (1988). «Gifts as Economic Signals and Social Symbols». American Journal of Sociology, 94, S180-S214.

[7] CARMICHAEL, H. L. y MACLEOD, W. B. (1997). «Gift giving and the evolution of cooperation». International Economic Review, 38, 485-509.

[8] COMISIÓN NACIONAL DE LA COMPETENCIA (2013). Informe sobre la aplicación de la guía de contratación y competencia a los procesos de licitación para la provisión de la sanidad pública en España. Madrid.

[9] EUROPEAN UNION (2013). Health and Economics Analysis for an Evaluation of the Public-Private Partnerships in Health Care Delivery across the EU. EAHC/2011/ Health/20. Recuperado en noviembre 2018 de https://ec.europa.eu/health/expert_panel/ sites/expertpanel/files/ppp_finalreport_en.pdf.

[10] FUNDACIÓ AVEDIS DONABEDIAN (2002). Avaluació de la reforma de l'atenció primària i de la diversificació de serveis. Barcelona, FAD.

[11] GODLEE, F. (2018). «Reinvigorating Cochrane». British Medical Journal, 362:k3966.

[12] GØTZSCHE, P. C. e IOANNIDIS, J. P. (2012). «Content area experts as authors: helpful or harmful for systematic reviews and meta-analyses?». British Medical Journal, 345:e7031.

[13] GRUP D'ETICA SOCIETAT CATALANA DE MEDICINA FAMILIAR I COMUNITARIA (2004). «La ética en la relación con la industria farmacéutica. Encuesta de opinión a médicos de familia en Cataluña». Atención Primaria, 34, 6-12.

[14] IASIST (2013). Evaluación de resultados de los hospitales en España según su modelo de gestión, Barcelona.

[15] IDIS (2018). Sanidad privada, aportando valor Análisis de situación 2018, Instituto para el Desarrollo e Integración de la Sanidad, Madrid.

[16] INSTITUT D'ESTUDIS DE LA SALUT (2006). Avaluació dels models de provisió de serveis d'atenció primària a Catalunya, Generalitat de Catalunya, Departament de Salut.

[17] INSTITUTE OF MEDICINE (2011). Clinical Practice Guidelines We Can Trust, Washington D.C., National Academies Press. 
[18] IOANNIDIS, J. P. A. (2015). «Cómo hacer más fiable la investigación que se publica». Revista Cubana de Información en Ciencias de la Salud, 26, 187-200.

[19] JØRGENSEN, A. W.; HILDEN, J. y GØTZSCHE, P. C. (2006). «Cochrane reviews compared with industry supported meta-analyses and other meta-analyses of the same drugs: systematic review». British Medical Journal, 333, 782.

[20] JOSIAH MACY JUNIOR FOUNDATION (2008). Continuing education in the health professions: improving healthcare through lifelong learning 2008. Recuperado en noviembre 2018 de http://macyfoundation.org/docs/macy_pubs/pub_ContEd_inHealthProf.pdf.

[21] KATZ, H. P.; GOLDFINGER, S. E. y FLETCHER, S. W. (2002). «Academia-industry collaboration in continuing medical education: Description of two approaches». Journal of Continuing Education in the Health Professions, 22, 43-54.

[22] KELMAN, S. J. (2002). «Contracting». En M. Lester y Salamon (eds.), The Tools of Government: A Guide to the New Governance. Nueva York, Oxford University Press, 282-318.

[23] KRUSE, F. M.; STADHOUDERS, N. W.; ADANG, E. M.; GROENEWOUD, S. y JEURISSEN, P. P. T. (2018). «Do private hospitals outperform public hospitals regarding efficiency, accessibility, and quality of care in the European Union? A literature review». International Journal of Health Planning and Management, 33 (2), e434-e453.

[24] LONSDALE, C. (2005). «Risk Transfer and the UK Private Finance Initiative: A Theoretical Analysis». Policy and Politics, 33 (2), 231-249.

[25] LÓPEZ-CASASNOVAS, G. y DEL LLANO, J. E. (eds.) (2017). Colaboración público-privada en sanidad: el modelo Alzira. Fundación Gaspar Casal. Madrid.

[26] LÜSCHER, T. F. (2012). «Good publishing practice». European Heart Journal, 33 (5), 557-561.

[27] MACHADO GUIMARÃES, C.y CRESPO DE CARVALHO, J. (2011). «Outsourcing in the Healthcare Sector-A State-of-the-Art Review». Supply Chain Forum, 12 (2), 138-146.

[28] MENÉNDEZ, A. (2008). «La gestión indirecta de la asistencia sanitaria pública. Reflexiones en torno al debate sobre la privatización de la sanidad». Revista de Administración Sanitaria Siglo XXI, 6 (2), 269-296.

[29] MENEU, R. (2010). «La promoción de medicamentos, apuntes para una mejor comprensión». Formación Médica Continuada en Atención Primaria, 17 (2), 53-55.

[30] NO GRACIAS (2018). Alianza para el buen gobierno del conocimiento biomédico en el sistema nacional de salud. Recuperado en noviembre 2018 de http://www.nogracias. eu/2018/11/03/alianza-buen-gobierno-del-conocimiento-biomedico-sistema-nacional-salud/.

[31] NOVOA, A. (2015). Veinte principios para la innovación y el buen gobierno del conocimiento biomédico. Recuperado en noviembre 2018 de http://www.nogracias. eu/2015/02/21/veinte-principio-innovacion-buen-gobierno-conocimiento-biomedico/.

[32] PEIRÓ, S. (2009). «Médicos e industra farmacéutica. Para estar así, mejor casados». Revista de Calidad Asistencial, 24 (2), 47-50.

[33] PEIRÓ, S.; MENEU, R.; ROSELLÓ, M. y TOBED, M. (1993). «Pago prospectivo por caso y duración de la estancia en hospitales privados concertados». Medicina Clínica (Barc.), 100, 372-374.

[34] PÉREZ-ROMERO, C.; ORTEGA-DÍAZ, M.I.; OCAÑA-RIOLA, R. y MARTÍN-MARTÍN J. J. (2017). «Análisis multinivel de la eficiencia técnica de los hospitales del 
Sistema Nacional de Salud español por tipo de propiedad y gestión». Gaceta Sanitaria, https://doi.org/10.1016/j.gaceta.2018.02.005.

[35] RELMAN, A. S. (2001). «Separating Continuing Medical Education From Pharmaceutical Marketing». JAMA, 285 (15), 2009-2012.

[36] REPULLO, J. R. (2008). «Externalización, eficiencia y calidad (primera parte). Público y privado en la sanidad». Revista de Calidad Asistencial, 23 (2), 83-87.

[37] ROIG, F. y BORREGO, A. (2017). «Declaraciones de conflictos de interés en las publicaciones biomédicas. Estudio de cuatro revistas clínicas españolas». Acta Bioethica, 23 (1), 55-62.

[38] SAITO, S.; MUKOHARA, K. y MIYATA, Y. (2014). «Chronological changes in Japanese physicians' attitude and behavior concerning relationships with pharmaceutical representatives: a qualitative study». PloS One, 9:e106586.

[39] SAVAS, E. S. (1990). «A Taxonomy of Privatization Strategies». Policy Studies Journal, 18 (2), 343-355.

[40] SÍNDIC DE GREUGES (2002). «Informe extraordinari del Síndic de Greuges al Parlament de Catalunya sobre l'aproximació a la situació de l'atenció primària de salut a Catalunya». Butlletí Oficial del Parlament de Catalunya, 24 de octubre de 2002.

[41] SINDICATURA DE COMPTES (2018). Auditoría operativa de la concesión de la asistencia sanitaria integral en el Departamento de Salud de Torrevieja, Valencia. Recuperado en noviembre 2018 de https://www.sindicom.gva.es/web/informes.nsf/0/14C99D556CDEBC1EC125833C0030F2E2/\$file/INF-2016-DSTORREVIEJA-09-Final2018-10-29-signat.pdf.

[42] SONDERGAARD, J.; VACH, K.; KRAGSTRUP, J. y ANDERSEN. M. (2009). «Impact of pharmaceutical representative visits on GPs' drug preferences». Family Practice, 26, 204-209.

[43] SPITHOFF, S. (2014). «Industry involvement in continuing medical education. Time to say no». Canadian Family Physician, 60 (8), 694-696.

[44] STAMATAKIS,E.; WEILER, R.e IOANNIDIS, J. P. (2013). «Undue industry influences that distort healthcare research, strategy, expenditure and practice: a review». European Journal of Clinical Investigation, 43, 469-475.

[45] STEINMAN, M. A.; SHLIPAK, M. G. y MCPHEE, S. J. (2001). «Of principles and pens: attitudes and practices of medicine housestaff toward pharmaceutical industry promotions». American Journal of Medicine, 110, 551-557.

[46] TYNKKYNEN, L. K. y VRANGBÆK, K. (2018). «Comparing public and private providers: a scoping review of hospital services in Europe". BMC Health Services Research, 18(1):141.

[47] VECCHI, V. y HELLOWELL, M. (eds.) (2018). Public-Private Partnerships in Health. Improving Infrastructure and Technology. Palgrave Macmillan. Cham, Switzerland.

[48] WAYANT, C.; TURNER, E. y MEYER, C. et al. (2018). «Financial Conflicts of Interest Among Oncologist Authors of Reports of Clinical Drug Trials». JAMA Oncol., 4 (10), 1426-1428.

[49] WILLIAMSON, O. (1990). «Transaction Cost Economics and Organisation Theory». En O. Williamson (ed.), Organisation Theory: From Chester Barnard to the Present and Beyond. Oxford University Press. New York. 